\title{
Sex differences in risk and heritability estimates on primary knee osteoarthritis leading to total knee arthroplasty: a nationwide population based follow up study in Danish twins
}

Søren Glud Skousgaard ${ }^{1,2,3^{*}}$ (D), Axel Skytthe ${ }^{4}$, Sören Möller ${ }^{4}$, Søren Overgaard ${ }^{2,3}$ and Lars Peter Andreas Brandt ${ }^{1,3}$

\begin{abstract}
Background: Symptomatic knee osteoarthritis is a highly age and sex associated complex disease. Little is known about the causes behind this age and sex associated increase, or if genetic and environmental factors impacts differently by gender. Our study examined the risk and heritability of primary knee osteoarthritis leading to total knee arthroplasty and whether these differences were attributable to sex and age differences in heritability.

Methods: All twins of known zygosity from The Danish Twin Register alive in 1997 were examined in a nationwide population based follow-up study collecting information on all twins recorded in The Danish Knee Arthroplasty from 1997 to follow-up in 2010. Our main outcomes were the cumulative incidence, probandwise concordance rates, heritability, within pair correlations in monozygotic and dizygotic twin pairs and the genetic and environmental influence estimated in models taking into account that individuals may not have had a total knee arthroplasty at follow up.

Results: 92,748 twins were eligible for analyses and 576 twins had a record of primary knee osteoarthritis in The Danish Knee Arthroplasty Register at follow-up comprising 358 female and 218 male twin cases. The risk increased particular after the age of 50 years displaying significant sex differences in the elderly. In the sex stratified analyses a discrete genetic component was found in females, but in males no genetic component could be detected. In both genders common and unique environmental factors were highly significant. In the sex-adjusted analysis an additive genetic component of $18 \%(0 ; 62)$, a shared environmental component of $61 \%(25 ; 97)$ and an individual environmental component of $21 \%(6 ; 36)$ accounted for the variation in liability to primary total knee arthroplasty.

Conclusion: The risk of primary total knee arthroplasty increases significantly after the age of 50 years, in particular in females, displaying significant sex differences in the elderly. After sex-adjustment $82 \%$ of the variation in liability to primary total knee arthroplasty was attributable to common and unique environmental factors; the remaining $18 \%$ of this variation was attributable to additive genetic factors indicating a pivotal impact of environmental factors on this disease.
\end{abstract}

Keywords: Knee osteoarthritis, Cumulative incidence, Twin study, Heritability, Total knee arthroplasty

\footnotetext{
* Correspondence: soeren.skousgaard@rsyd.dk

'Department of Occupational and Environmental Medicine, Odense

University Hospital, 5000 Odense C, Denmark

${ }^{2}$ Department of Orthopaedic Surgery and Traumatology \& Orthopedic

Research Unit, Institute of Clinical Research, University of Southern Denmark,

5000 Odense C, Denmark

Full list of author information is available at the end of the article
} 


\section{Background}

Osteoarthritis (OA) is the most frequent joint disorder in western societies, and will presumably develop into one of the top-ranking causes of physical functional impairment and disability, including work-related disorders and absenteeism, during the next one or two decades [1-3]. Knee OA is a highly age and sex-associated prevalent and complex disease, with a substantial impact on the quality of life from pain and physical immobility, and consequently a significant limitation in social and working life [4]. Additionally, the co-morbidity associated with symptomatic knee OA is subject to concern because in a recent study an increased all-cause and disease-specific mortality has been reported in patients with symptomatic and radiographic hip and knee OA [5]. The prevalence of radiographic knee OA from the age of 50 years onwards ranges between 20 and $44 \%$, higher in females than in males [6-8]. However, the lifetime risk of symptomatic knee OA has recently been reported to be as high as $46.8 \%$ and $39.8 \%$ in females and males, respectively; in obese individuals, the lifetime risk of symptomatic knee OA was reported to be high as $60.5 \%$ [9].

Knee OA is a complex and multifactorial disease caused by environmental and genetic factors. Environmental risk factors frequently referred to include body mass index (BMI) $[10,11]$, previous knee injury [12-14] and kneestraining work tasks $[15,16]$. However, few studies into the genetic influence on knee OA have been published. A moderate genetic contribution to radiographic knee OA in healthy female twins has been reported in two crosssectional studies with heritability estimates of $39 \%$ and $37 \%$ respectively $[17,18]$. Additionally, the progression of radiographic knee OA in females has previously been reported to be under genetic influence [19]. These studies, however, examined non-symptomatic radiographic knee OA in females only. Consequently, little is known of the genetic influence in males, or whether genetic factors affect males and females differently or, indeed, whether genetic factors differ between radiographic and symptomatic knee OA. In a previously published sibling study including both genders listed for total knee arthroplasty (TKA), an increased risk in siblings to index cases after adjustment for sex, age, knee pain, BMI, Heberden's nodes and meniscectomy was reported with odds ratios of 2.9 and 1.7 for tibiofemoral and patellofemoral OA, respectively [20]. In a previously published twin study, based on self-reported physician-diagnosed OA, the authors could not detect a genetic influence on OA (finger, hip, knee and ankle) in males, leaving common and unique environmental factors as the best model fit; however, in women an additive genetic component of $44 \%$ in OA liability (finger, hip, knee and ankle) was reported [21]. However, the issue of OA in different joints being caused by common genetic processes or the genetic influence being joint site specific has previously been stressed as highly important for the design of future studies examining the nature of the genetic contribution to OA [18].

Studies defining knee OA cases from radiographic findings with or without symptoms may encounter some difficulties in defining their cases because disease severity varies and the correlation between clinical presentation and radiographic findings in OA generally is inconsistent $[6-8,22]$. For reasons already mentioned we decided to use a twin who had undergone a TKA as a case, because this case definition represents a well-defined outcome and a heavy and significant disease burden contrary to cases based on conventional radiographic examination [22].

Accordingly, the objectives of this study were to examine the probability and heritability of primary OA of the knee leading to TKA, both sex stratified and sex adjusted, in a competing risk setting by means of the cumulative incidence function (CIF), biometric modelling, the agerelated proband-wise concordance rates and cumulative heritability.

\section{Methods}

Our study participants were selected from the Danish Twin Register (DTR). The DTR was established in 1954 and comprises more than 170,000 twins born in Denmark since 1870. The completeness of the twin ascertainment is high; after adjustment for infant mortality, $90 \%$ of the twins born before and up to 1968 have been ascertained, with complete ascertainment of all live-born twins since 1968 [23, 24]. Zygosity of same-sex twins is assessed by a four-item questionnaire on the similarity of the two twins, which will classify their zygosity correctly in $95 \%$ of all same-sex twin pairs [25]. All twins in the DTR alive at 1 January 1997 comprised the study cohort. Information on sex, date of birth, zygosity and vital status was used. The Danish Knee Arthroplasty Register (DKR) is a nationwide population-based health register established in 1997 and holds information on TKA surgery performed on a national basis. The register holds information on sex, date of birth, the WHO International Classification of Diseases version 10 (ICD10) diagnosis, being that which indicated the surgical treatment, and the date of surgery. The completeness of registration is 90-92\% based on annual reports and thus the DKR provides a sound basis for large population-based epidemiological studies [26, 27]. Using the unique Danish personal identification number (CPR) as a key for linkage, all Danish twins with a TKA and ICD10 diagnoses of M170 and M171 for the period of 1 January 1997-1 December 2010 were identified (Fig. 1). Information on sex, date of birth, the ICD10 diagnoses M170 and M171, and the date of surgery from the period of 1 January 1997-1 December 2010 were used. Our final study cohort comprised monozygotic (MZ), same- 


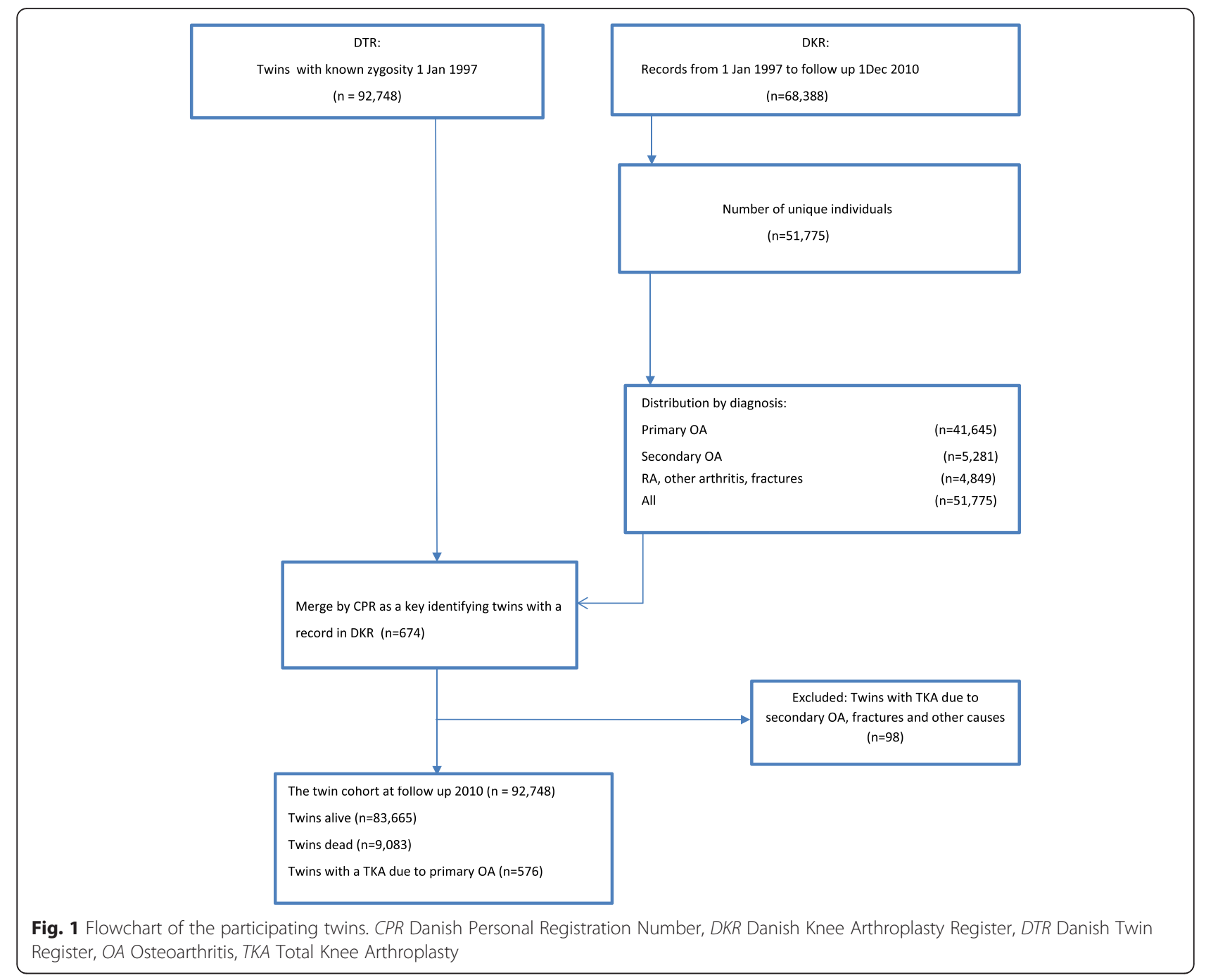

sex dizygotic (SS-DZ) and opposite-sex dizygotic (OS-DZ) twins, and a case was defined as a twin who had undergone a TKA due to primary knee OA independent of co-twin status.

\section{Ethical approval}

The study was reviewed and approved by The Regional Research Ethical Committees of Southern Denmark and The Danish Data Protection Agency, and permission was granted to use the relevant data from the Danish Hip Arthroplasty Register and the DTR.

\section{Statistical analyses}

Our objectives were to estimate the probability and heritability of TKA due to primary knee OA. To challenge these aims we implemented a time-to-event methodology in analysing our twin data taking into account the presence of right censoring and the competing risk of death in the study population.
In the classical twin design (CTD), MZ twins are assumed to have identical genotypes, whereas DZ twins on average share one-half of their segregating genes as ordinary siblings. If a greater phenotypic similarity in $\mathrm{MZ}$ twins compared with that of $\mathrm{DZ}$ twins is observed, a genetic influence on the disease in question can be inferred [28]. The similarity in MZ and DZ twin pairs with a TKA was assessed by means of case-wise and proband-wise concordance rates and tetrachoric correlation coefficients. These concordance rates reflect the probability of one twin having the disease in question conditional that the co-twin is affected, and when all concordant pairs are doubly ascertained the proband-wise concordance rate equals that of the case-wise concordance rate [28]. The impact of environmental and genetic effects on a specific disease is reflected in the twin resemblance for liability to the disease expressed as correlations. In the case of a dichotomous outcome this resemblance is expressed as tetrachoric correlations and is estimated 
under the multifactorial threshold model assumption, which assumes that there is an underlying normally distributed liability to disease. The disease becomes manifest when an individual exceeds a threshold on the liability distribution corresponding to the overall prevalence of the disease $[28,29]$. The threshold model is graphically displayed in Fig. 2.

Variation of a trait in a twin population can be separated into genetic factors, additive genetic (A) and dominant genetic (D), and environmental factors, shared environment $(\mathrm{C})$ and unique environment (E) to the individual. Models containing combinations of these variance components can be constructed based on the known underlying correlation structure in a twin population [28].

To estimate the probability or risk of TKA we implemented the CIF, which takes the occurrence of competing risks into account [30-32]. If an individual is at risk of experiencing more than one event, each affecting the other, these events are called competing risks. In a population of older individuals with a long-term follow-up, the risk of knee OA leading to TKA and death become competing risks. We used the CIF to estimate the probability of undergoing a TKA because of knee OA adjusted for the competing risk of death by means of the Aalen-Johansen estimator. This method is based on the Markov illness death model with states 'healthy', 'diseased' and 'dead', where the transition probabilities describe the probability that a healthy individual at a later time will be diseased or dead [33].

Bivariate probit modelling in twin data is a new approach for analysing genetic and environmental factors on knee OA leading to TKA, and takes into account the presence of right censoring and competing risks in the data [34]. We implemented a weighted time regression analysis based on the inverse probability weighting (IPW)

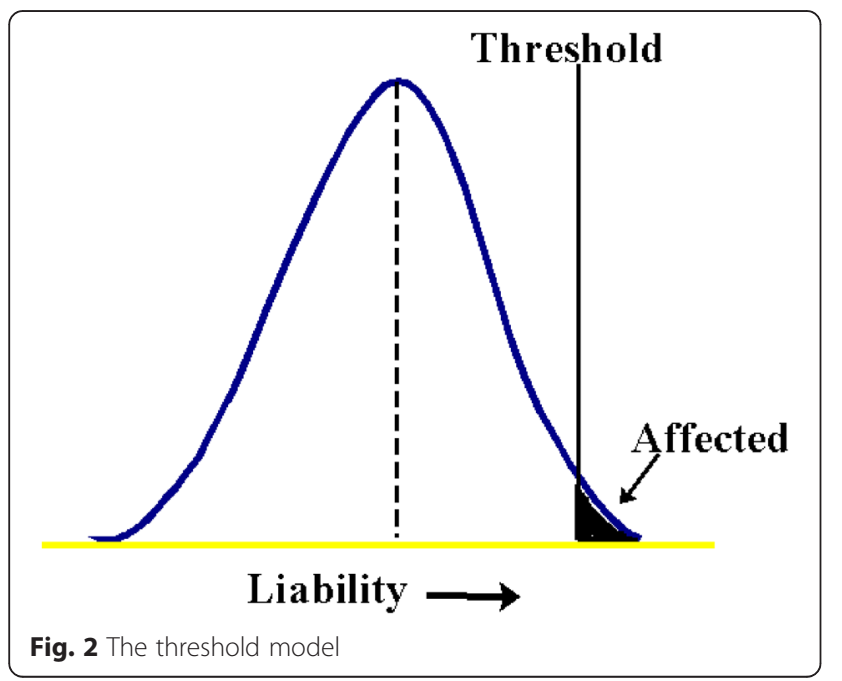

technique which is used to account for unbalanced sampling probabilities; weighting by the inverse of the probability of being sampled allows each observation to statistically represent the sampled non-case individuals as cases in the population [35, 36].

In a time-to-event analysis with competing risks, only a proportion of the cases are known at follow-up because individuals appearing as non-cases may become cases prospectively at a time we do not know, or are lost to follow-up. This is termed right censoring and we used liability-threshold modelling with IPW to analyse the twin data [29, 34-36].

Our biometric model fitting included saturated models and models composed of the variance components ACE, $\mathrm{ADE}, \mathrm{AE}, \mathrm{DE}$ and $\mathrm{CE}$, both sex stratified and sex adjusted. For comparisons between models, a log likelihood ratio test and Akaike's Information Criterion (AIC) were used. However, we also included the broad sense heritability based on the polygenetic liability-threshold model, which is a measure of the proportion of the variance in liability to a disease caused by additive and non-additive genetic effects [29].

The analyses were carried out in bivariate probit models for twin data implemented in the R Mets package (https:// cran.r-project.org/web/packages/mets/index.html) and using the methods of estimating the proband-wise concordance function for competing risks data [37]. For comparisons between groups, a two-tailed $t$ test or chi-square test was used as appropriate. $p \leq 0.05$ was considered significant, and confidence intervals (CIs) were expressed as the $95 \% \mathrm{CI}$. Calculations were carried out in the statistical software $\mathrm{R}$ and Stata11 (StataCorp LP, 4905 Lakeway Drive, College Station, Texas 77845-4512, USA).

\section{Results}

Figure 1 displays the flow of the participating twins. The twin cohort comprised 92,748 twins, with 674 twins recorded in the DKR of which 576 had undergone a TKA due to primary knee OA at follow-up. Table 1 presents summary statistics from the two registers.

\section{Tetrachoric correlations}

Table 2 presents tetrachoric correlations in the same-sex and opposite-sex twin pairs where both twins were alive at entry. We found no significant differences between male MZ and DZ twin pairs contrary to female MZ and DZ twin pairs, indicating a lack of genetic component in males but detectable in females. The test for within-pair independence for TKA in MZ males is borderline significant $(p=0.05)$ compared with that of $\mathrm{DZ}$ male pairs $(p<0.0001)$, further indicating that a genetic influence in males may be rather insignificant. However, the correlation in DZ male pairs was markedly higher than that of 
Table 1 Distribution of TKA by register, zygosity and sex

\begin{tabular}{lll}
\hline Distribution by register, sex and TKA & DTR & DKR \\
Number & 92,748 & 51,775 \\
Females & 46,171 & 32,076 \\
Males & 46,577 & 19,699 \\
Record in DKR & $674^{\mathrm{a}}(85)$ & $41,645(81)$ \\
$\quad$ Females & $349(61)$ & $26,719(62)$ \\
Males & 227 & 15,111 \\
Mean age (years) & $67.1(39-94)$ & $67.6(17-100)$ \\
Females & $67.7(40-94)$ & $68.3(17-100)$ \\
$\quad$ Males & $66.3(39-88)$ & $66.3(21-96)$ \\
Distribution by zygosity, sex and TKA & Males & Females \\
MZ with TKA & 39 & $82(68)$ \\
MZ without TKA & 9,739 & $10,135(51)$ \\
DZ with TKA & 103 & $157(60)$ \\
DZ without TKA & 17,727 & $16,691(48)$ \\
OS with TKA & 85 & $110(56)$ \\
OS without TKA & 18,884 & $18,996(50)$ \\
Total & 46,577 & $46,171(50)$ \\
\hline
\end{tabular}

Data presented as number, number (percentage) or mean (range)

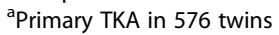

DKR Danish Knee Arthroplasty Register, DTR Danish Twin Register, DZ dizygotic, MZ monozygotic, OS opposite sex, TKA total knee arthroplasty

the $\mathrm{MZ}$ male pairs, indicating a significant influence from common environmental factors in males.

\section{Cumulative incidence function}

Figure 3 shows the cumulative incidence for TKA due to primary knee OA. An increasing risk was found from the age of 50 years in both genders; peaking to $2.5 / 100$ individuals in males and 4/100 individuals in females at 85 years of age.

\section{Proband-wise concordance rates on age}

In Fig. 4 the proband-wise concordance rates including censoring are displayed separately for men and women. In males, no difference in MZ and DZ twin pairs was observed; indicating that the genetic influence in males was not detectable or present. In females, a MZ/DZ twin pair difference is closing in by age, indicating a decreasing genetic influence in females by age; however the $95 \%$ CIs did overlap.

\section{Prevalences, tetrachoric correlations and case-wise concordance rates}

Equal prevalence in MZ and DZ twin pairs in all models was observed; however, in males the saturated model (all co-variances are treated as free parameters) did not support the existence of a genetic component, as both the case-wise concordance rates and tetrachoric correlations did not differ between MZ and DZ twin pairs. In females

Table 2 Concordant and disconcordant pairs and tetrachoric correlations by zygosity in twin pairs where both twins in the pair were alive at entry

\begin{tabular}{|c|c|c|c|c|}
\hline Zygosity & $\begin{array}{l}\text { Concordant TKA due } \\
\text { to OA pairs }(n)\end{array}$ & $\begin{array}{l}\text { Discordant TKA due } \\
\text { to OA pairs }(n)\end{array}$ & $\begin{array}{l}\text { Concordant pairs without } \\
\text { OA pairs }(n)\end{array}$ & $\begin{array}{l}\text { Tetrachoric correlation } \\
\text { (rho) }\left(p \text { value }^{\mathrm{a}}\right)\end{array}$ \\
\hline MZ males & 1 & 29 & 4509 & $0.45(0.05)$ \\
\hline DZ males & 4 & 72 & 8176 & $0.51(<0.0001)$ \\
\hline MZ females & 10 & 52 & 4701 & $0.73(<0.0001)$ \\
\hline DZ females & 6 & 122 & 7575 & $0.42(<0.0001)$ \\
\hline OS-DZ & 3 & 158 & 17,490 & $0.36(0.004)$ \\
\hline
\end{tabular}

aTest of within-pair independence for TKA due to OA

$D Z$ dizygotic, MZ monozygotic, OA osteoarthritis, OS-DZ opposite-sex dizygotic, rho tetrachoric correlation coefficient, TKA total knee arthroplasty 


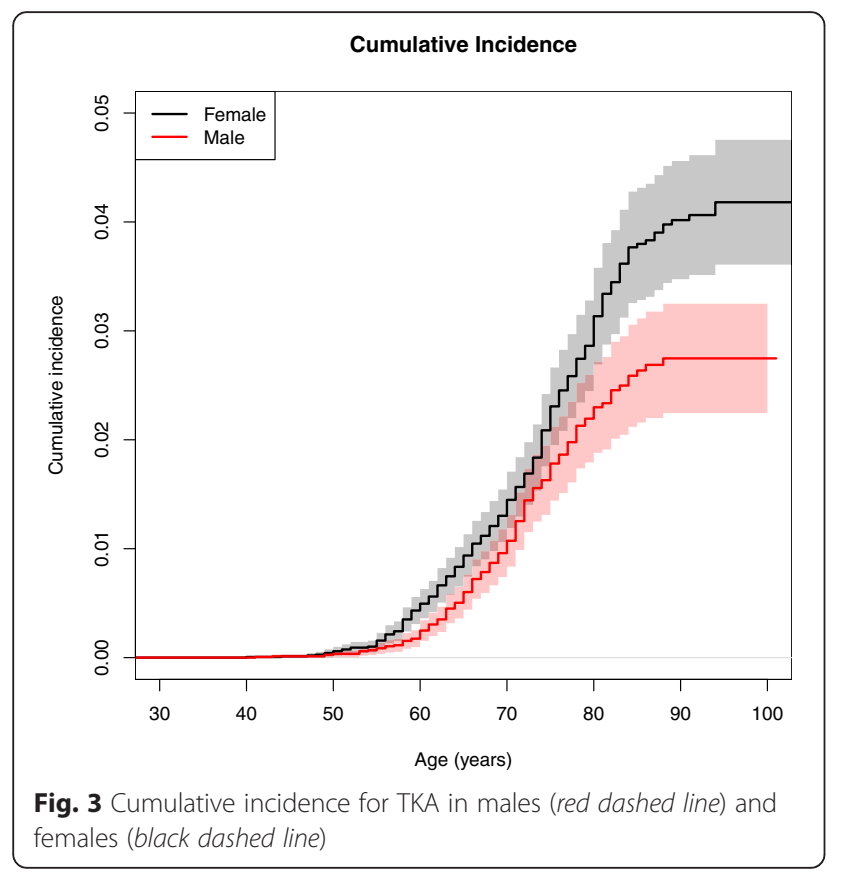

this difference was detectable, but not significant, albeit favouring a broad sense heritability of $6 \%(0 ; 73)$. In the sex-adjusted saturated model the differences in case-wise concordance rates and tetrachoric correlations coefficients between MZ and DZ twin pairs were notable, favouring a broad sense heritability of $18 \%(0 ; 62)$ (see Tables 3 , 4 and 5).

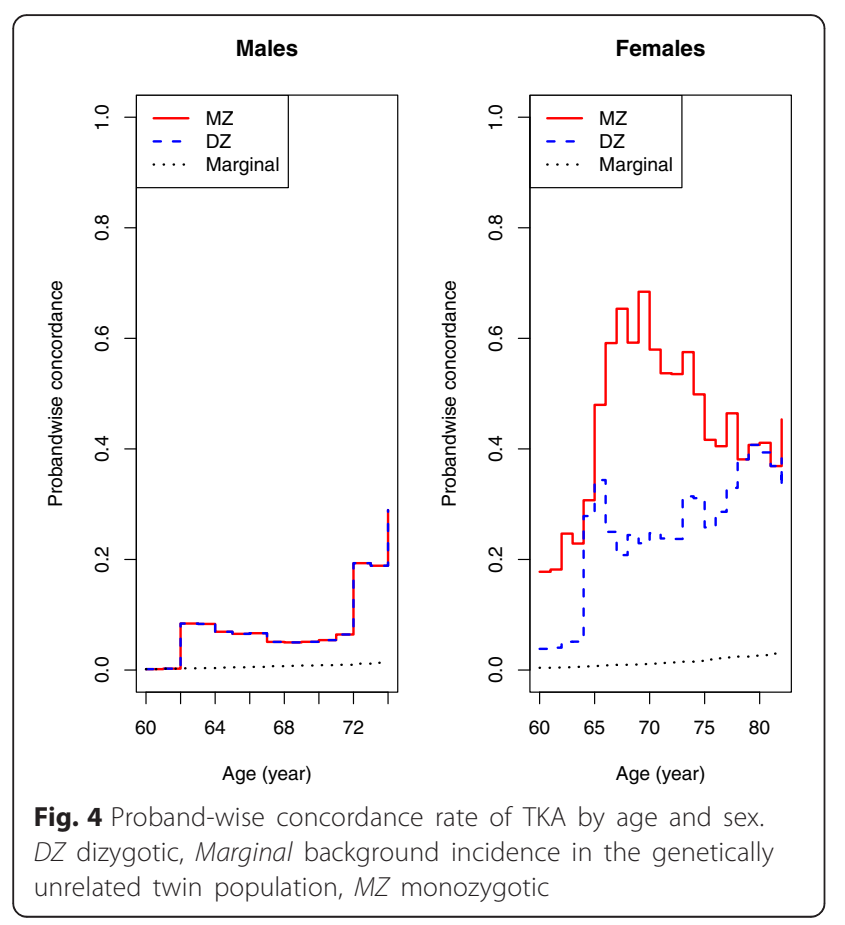

\section{Biometric modelling}

The first model in Tables 3, 4 and 5 displays the broad sense heritability estimates reflecting the proportion of the variance attributable to genetic factors. In males, the best fitting model was the $\mathrm{CE}$ model of common and individual environmental factors; fixing $\mathrm{A}$ at 0 in the ACE model did not change the fit at all $(p=1.0)$ and the CE gave the best fit by the AIC. In females, the ACE model provided the best fit to the data; setting A at 0 in the ACE model did not produce a significantly worse fit $(p=0.61)$, but the ACE model gave the best fit by the AIC. In the sex-adjusted saturated model the $\mathrm{DZ}$ correlation was markedly larger than half that of the MZ correlation, underlining the presence of a common environmental component. Fixing $\mathrm{C}$ at 0 in the sex-adjusted ACE model produced a significantly worse fit in the AE model $(p<0.0001)$. Setting A at 0 in the sex-adjusted ACE model produced a significant worse fit in the CE model $(p=$ 0.05 ). Excluding a genetic component by comparing the $\mathrm{CE}$ with the saturated model likewise produced a significant worse fit $(p=0.05)$ and the sex-adjusted ACE model gave the best fit comparing the alternative models $\mathrm{AE}$, $\mathrm{ADE}, \mathrm{DE}$ and $\mathrm{CE}$ by the AIC. This model produced an additive genetic component of $18 \%(0 ; 62)$ and a highly significant common environmental component of $61 \%$ $(25 ; 97)$, with a unique environmental component accounting for $21 \%(6 ; 36)$ of the population liability for TKA due to primary knee OA.

Both the sex-stratified and sex-adjusted best model fit provided ambient evidence of a highly significant common environmental influence. Indeed, the observed difference in DZ-MZ correlations-that is, the DZ correlation was markedly larger than half that of the MZ correlationstrengthened and emphasised the presence of a common environmental component favouring the $\mathrm{ACE}$ and $\mathrm{CE}$ models in females and males, respectively.

\section{Heritability on age}

The age-associated changes in the variance components in the CE and ACE models are displayed in Fig. 5 for males and in Figs. 6 and 7 for females, respectively. In males, the common and individual environmental influences remain high from 60 to 70 years of age. In females, the genetic influence decreased with increasing age to disappear at age around 80 years. In Fig. 7 the heritability on age in females is displayed with an estimate of $40 \%$ at age 65-66 years.

\section{Discussion}

To our knowledge this is the first twin study of primary knee OA leading to TKA, including both sexes, as well as the same-sex and opposite-sex twin pairs. We could not estimate a genetic component in males, inferring knee OA in males as primarily an environmental disease. 
Table 3 Heritability and biometric modelling in males with knee OA

\begin{tabular}{|c|c|c|c|c|c|c|c|c|c|c|c|c|c|c|c|c|}
\hline \multirow{2}{*}{$\begin{array}{l}\text { Model in } \\
\text { males }\end{array}$} & \multirow{2}{*}{$\begin{array}{l}\text { MZ } \\
\text { prevalence }\end{array}$} & \multirow{2}{*}{$\begin{array}{l}\text { DZ } \\
\text { prevalence }\end{array}$} & \multirow[t]{2}{*}{ MZ rho } & \multirow[t]{2}{*}{ DZ rho } & \multirow[t]{2}{*}{$\mathrm{MZ}$ cwc } & \multirow[t]{2}{*}{ DZ cwe } & \multirow[t]{2}{*}{$\mathrm{H}$} & \multicolumn{4}{|c|}{ Variance component estimates } & \multicolumn{4}{|c|}{ Chi-square test statistics } & \multirow[b]{2}{*}{ AIC } \\
\hline & & & & & & & & A & $\mathrm{D}$ & $C$ & E & $\mathrm{LL}$ & $-2 \ln$ & $\mathrm{df}$ & $p$ value & \\
\hline Sat & $\begin{array}{l}0.02 \\
(0.01-0.02)\end{array}$ & $\begin{array}{l}0.02 \\
(0.01-0.02)\end{array}$ & $\begin{array}{l}0.66 \\
(0.03-0.92)\end{array}$ & $\begin{array}{l}0.71 \\
(0.39-0.87)\end{array}$ & $\begin{array}{l}0.26 \\
(0.05-0.70)\end{array}$ & $\begin{array}{l}0.30 \\
(0.13-0.55)\end{array}$ & $\begin{array}{l}0 \\
(0.00-0.89)\end{array}$ & - & - & - & - & -3387.1 & 0.021 & 1 & $\begin{array}{l}\text { Compare CE, } \\
p=0.65\end{array}$ & 6780.14 \\
\hline ACE & $\begin{array}{l}0.02 \\
(0.01-0.02)\end{array}$ & $\begin{array}{l}0.02 \\
(0.01-0.02)\end{array}$ & $\begin{array}{l}0.7 \\
(0.42-0.85)\end{array}$ & $\begin{array}{l}0.7 \\
(0.42-0.85)\end{array}$ & $\begin{array}{l}0.29 \\
(0.14-0.51)\end{array}$ & $\begin{array}{l}0.29 \\
(0.14-0.51)\end{array}$ & 0 & 0 & - & $\begin{array}{l}0.69 \\
(0.48-0.90)\end{array}$ & $\begin{array}{l}0.31 \\
(0.1-0.52)\end{array}$ & -3387.2 & 25.8 & 1 & $\begin{array}{l}\text { Compared AE, } \\
p<0.0001\end{array}$ & 6780.35 \\
\hline ADE & $\begin{array}{l}0.02 \\
(0.01-0.02)\end{array}$ & $\begin{array}{l}0.02 \\
(0.01-0.02)\end{array}$ & $\begin{array}{l}0.79 \\
(0.45-0.93)\end{array}$ & $\begin{array}{l}0.39 \\
(0.28-0.50)\end{array}$ & $\begin{array}{l}0.39 \\
(0.18-0.66)\end{array}$ & $\begin{array}{l}0.10 \\
(0.07-0.16)\end{array}$ & $\begin{array}{l}0.79 \\
(0.57-1.0)\end{array}$ & $\begin{array}{l}0.79 \\
(0.57-1.0)\end{array}$ & 0 & - & $\begin{array}{l}0.21 \\
(0.0-0.43)\end{array}$ & -3400.1 & 0 & 1 & $\begin{array}{l}\text { Compare DE, } \\
p=1.0\end{array}$ & 6806.14 \\
\hline $\mathrm{AE}$ & $\begin{array}{l}0.02 \\
(0.01-0.02)\end{array}$ & $\begin{array}{l}0.02 \\
(0.01-0.02)\end{array}$ & $\begin{array}{l}0.79 \\
(0.45-0.93)\end{array}$ & $\begin{array}{l}0.39 \\
(0.28-0.50)\end{array}$ & $\begin{array}{l}0.39 \\
(0.18-0.66)\end{array}$ & $\begin{array}{l}0.10 \\
(0.07-0.16)\end{array}$ & $\begin{array}{l}0.79 \\
(0.57-1.0)\end{array}$ & $\begin{array}{l}0.79 \\
(0.57-1.0)\end{array}$ & - & - & $\begin{array}{l}0.21 \\
(0.0-0.43)\end{array}$ & -3400.1 & 25.8 & 1 & $\begin{array}{l}\text { Compare Sat, } \\
p<0.0001\end{array}$ & 6804.14 \\
\hline CE & $\begin{array}{l}0.02 \\
(0.01-0.02)\end{array}$ & $\begin{array}{l}0.02 \\
(0.01-0.02)\end{array}$ & $\begin{array}{l}0.69 \\
(0.42-0.85)\end{array}$ & $\begin{array}{l}0.69 \\
(0.42-0.85)\end{array}$ & $\begin{array}{l}0.29 \\
(0.14-0.51)\end{array}$ & $\begin{array}{l}0.29 \\
(0.14-0.51)\end{array}$ & - & - & - & $\begin{array}{l}0.69 \\
(0.48-0.90)\end{array}$ & $\begin{array}{l}0.31 \\
(0.1-0.52)\end{array}$ & -3387.2 & 0 & 1 & $\begin{array}{l}\text { Compare ACE, } \\
p=1.0\end{array}$ & 6778.35 \\
\hline DE & $\begin{array}{l}0.02 \\
(0.01-0.02)\end{array}$ & $\begin{array}{l}0.02 \\
(0.01-0.02)\end{array}$ & $\begin{array}{l}0.76 \\
(0.38-0.92)\end{array}$ & $\begin{array}{l}0.19 \\
(0.13-0.25)\end{array}$ & $\begin{array}{l}0.36 \\
(0.14-0.66)\end{array}$ & $\begin{array}{l}0.04 \\
(0.03-0.06)\end{array}$ & $\begin{array}{l}0.76 \\
(0.51-1.0)\end{array}$ & - & $\begin{array}{l}0.76 \\
(0.51-1.0)\end{array}$ & - & $\begin{array}{l}0.24 \\
(0.00-0.49)\end{array}$ & -3414.6 & 29.1 & 1 & $\begin{array}{l}\text { Compare ADE, } \\
P<0.0001\end{array}$ & 6833.24 \\
\hline
\end{tabular}

$A$ additive genetic, $A / C$ Akaike's Information Criterion, $C$ common environment, $c w C$ case-wise concordance rate, $D$ dominant genetic, $d f$ degrees of freedom, $D Z$ dizygotic, $E$ unique environment, $H$ broad sense heritability, $L L=\log$ likelihood of model, $-2 \mathrm{ln}=$ Likelihood Ratio chi-square test, $M Z$ monozygotic, rho tetrachoric correlation coefficient, Sat saturated model, $O A$ osteoarthritis

The CE model in bold displayes the best model fit by the AIC. Fixing the additive genetic component at zero produced no worse fit $(p=1.0)$ 
Table 4 Heritability and biometric modelling in females with knee OA

\begin{tabular}{|c|c|c|c|c|c|c|c|c|c|c|c|c|c|c|c|c|}
\hline \multirow{2}{*}{$\begin{array}{l}\text { Model in } \\
\text { females }\end{array}$} & \multirow{2}{*}{$\begin{array}{l}\text { MZ } \\
\text { prevalence }\end{array}$} & \multirow{2}{*}{$\begin{array}{l}\mathrm{DZ} \\
\text { prevalence }\end{array}$} & \multirow[t]{2}{*}{ MZ rho } & \multirow[t]{2}{*}{ DZ rho } & \multirow[t]{2}{*}{ MZ cwe } & \multirow[t]{2}{*}{ DZ cwc } & \multirow[t]{2}{*}{$\mathrm{H}$} & \multicolumn{4}{|c|}{ Variance component estimates } & \multicolumn{4}{|c|}{ Chi-square test statistics } & \multirow[b]{2}{*}{ AIC } \\
\hline & & & & & & & & $\bar{A}$ & $\mathrm{D}$ & $C$ & $E$ & $\overline{\mathrm{LL}}$ & $-2 \ln$ & $d f$ & $p$ value & \\
\hline Sat & $\begin{array}{l}0.03 \\
(0.03-0.04)\end{array}$ & $\begin{array}{l}0.03 \\
(0.03-0.04)\end{array}$ & $\begin{array}{l}0.77 \\
(0.53-0.90)\end{array}$ & $\begin{array}{l}0.74 \\
(0.48-0.88)\end{array}$ & $\begin{array}{l}0.42 \\
(0.25-0.62)\end{array}$ & $\begin{array}{l}0.39 \\
(0.22-0.60)\end{array}$ & $\begin{array}{l}0.06 \\
(0.0-0.58)\end{array}$ & - & - & - & - & -5877.6 & 34.2 & 1 & $\begin{array}{l}\text { Compare AE, } \\
p<0.0001\end{array}$ & $11,552.2$ \\
\hline ACE & $\begin{array}{l}0.03 \\
(0.03-0.04)\end{array}$ & $\begin{array}{l}0.03 \\
(0.03-0.04)\end{array}$ & $\begin{array}{l}0.77 \\
(0.53-0.90)\end{array}$ & $\begin{array}{l}0.74 \\
(0.48-0.88)\end{array}$ & $\begin{array}{l}0.42 \\
(0.25-0.62)\end{array}$ & $\begin{array}{l}0.39 \\
(0.22-0.60)\end{array}$ & $\begin{array}{l}0.06 \\
(0.0-0.58)\end{array}$ & $\begin{array}{l}0.06 \\
(0.0-0.58)\end{array}$ & - & $\begin{array}{l}0.71 \\
(0.29-1.00)\end{array}$ & $\begin{array}{l}0.23 \\
(0.06-0.41)\end{array}$ & -5877.6 & 34.2 & 1 & $\begin{array}{l}\text { Compare AE, } \\
p<0.0001\end{array}$ & $11,552.2$ \\
\hline $\mathrm{ADE}$ & $\begin{array}{l}0.03 \\
(0.03-0.04)\end{array}$ & $\begin{array}{l}0.03 \\
(0.03-0.04)\end{array}$ & $\begin{array}{l}0.80 \\
(0.62-0.91)\end{array}$ & $\begin{array}{l}0.40 \\
(0.33-0.47)\end{array}$ & $\begin{array}{l}0.46 \\
(0.30-0.63)\end{array}$ & $\begin{array}{l}0.15 \\
(0.12-0.19)\end{array}$ & $\begin{array}{l}0.80 \\
(0.67-0.94)\end{array}$ & $\begin{array}{l}0.80 \\
(0.67-0.94)\end{array}$ & 0 & - & $\begin{array}{l}0.20 \\
(0.06-0.33)\end{array}$ & -5894.8 & 0 & 1 & $\begin{array}{l}\text { Compare AE, } \\
p=1.0\end{array}$ & $11,575.6$ \\
\hline $\mathrm{AE}$ & $\begin{array}{l}0.03 \\
(0.03-0.04)\end{array}$ & $\begin{array}{l}0.03 \\
(0.03-0.04)\end{array}$ & $\begin{array}{l}0.80 \\
(0.62-0.91)\end{array}$ & $\begin{array}{l}0.40 \\
(0.33-0.47)\end{array}$ & $\begin{array}{l}0.46 \\
(0.30-0.63)\end{array}$ & $\begin{array}{l}0.15 \\
(0.12-0.19)\end{array}$ & $\begin{array}{l}0.80 \\
(0.67-0.94)\end{array}$ & $\begin{array}{l}0.80 \\
(0.67-0.94)\end{array}$ & - & - & $\begin{array}{l}0.20 \\
(0.06-0.33)\end{array}$ & -5894.8 & 23.3 & 1 & $\begin{array}{l}\text { Compare Sat, } \\
p<0.0001\end{array}$ & $11,573.6$ \\
\hline CE & $\begin{array}{l}0.03 \\
(0.03-0.04)\end{array}$ & $\begin{array}{l}0.03 \\
(0.03-0.04)\end{array}$ & $\begin{array}{l}0.76 \\
(0.60-0.86)\end{array}$ & $\begin{array}{l}0.76 \\
(0.60-0.86)\end{array}$ & $\begin{array}{l}0.41 \\
(0.28-0.55)\end{array}$ & $\begin{array}{l}0.41 \\
(0.28-0.55)\end{array}$ & - & - & - & $\begin{array}{l}0.76 \\
(0.63-0.88)\end{array}$ & $\begin{array}{l}0.24 \\
(0.12-0.37)\end{array}$ & -5877.7 & 0.26 & 1 & $\begin{array}{l}\text { Compare ACE, } \\
p=0.61\end{array}$ & $11,553.5$ \\
\hline DE & $\begin{array}{l}0.03 \\
(0.03-0.04)\end{array}$ & $\begin{array}{l}0.03 \\
(0.03-0.04)\end{array}$ & $\begin{array}{l}0.79 \\
(0.59-0.90)\end{array}$ & $\begin{array}{l}0.20 \\
(0.16-0.24)\end{array}$ & $\begin{array}{l}0.45 \\
(0.38-0.63)\end{array}$ & $\begin{array}{l}0.08 \\
(0.06-0.09)\end{array}$ & $\begin{array}{l}0.79 \\
(0.64-0.94)\end{array}$ & - & $\begin{array}{l}0.79 \\
(0.64-0.94)\end{array}$ & - & $\begin{array}{l}0.19 \\
(0.05-0.33)\end{array}$ & -5912.5 & 35.5 & 1 & $\begin{array}{l}\text { Compare ADE, } \\
p<0.0001\end{array}$ & $11,606.5$ \\
\hline
\end{tabular}

$A$ additive genetic, AIC Akaike's Information Criterion, $C$ common environment, $c w C$ case-wise concordance rate, $D$ dominant genetic, $d f$ degrees of freedom, $D Z$ dizygotic, $E$ unique environment, $H$ broad sense heritability, $L L=\log$ likelihood of model, $-2 \ln =$ Likelihood Ratio chi-square test, $M Z$ monozygotic, rho tetrachoric correlation coefficient, Sat saturated model, $O A$ osteoarthritis

The ACE model in bold displayes the best model fit by the AIC. Fixing the common environmental component C at zero produced a significantly worse fit $(p<0.0001)$ 
Table 5 Biometric models and chi-square test statistics, sex adjusted

\begin{tabular}{|c|c|c|c|c|c|c|c|c|c|c|c|c|c|c|c|c|}
\hline \multirow[t]{2}{*}{ Model } & \multirow{2}{*}{$\begin{array}{l}\text { MZ } \\
\text { prevalence }\end{array}$} & \multirow{2}{*}{$\begin{array}{l}\text { DZ } \\
\text { prevalence }\end{array}$} & \multirow[t]{2}{*}{ MZ rho } & \multirow[t]{2}{*}{ DZ rho } & \multirow[t]{2}{*}{ MZ cwe } & \multirow[t]{2}{*}{ DZ cwc } & \multirow[t]{2}{*}{$\mathrm{H}$} & \multicolumn{4}{|c|}{ Variance component estimates } & \multicolumn{4}{|c|}{ Chi-square test statistics } & \multirow[b]{2}{*}{$\mathrm{AIC}$} \\
\hline & & & & & & & & $\bar{A}$ & $\mathrm{D}$ & c & E & LL & $-2 \ln$ & df & $p$ value & \\
\hline Sat & $\begin{array}{l}0.03 \\
(0.03-0.04)\end{array}$ & $\begin{array}{l}0.03 \\
(0.03-0.04)\end{array}$ & $\begin{array}{l}0.79 \\
(0.59-0.90)\end{array}$ & $\begin{array}{l}0.70 \\
(0.50-0.83)\end{array}$ & $\begin{array}{l}0.44 \\
(0.28-0.62)\end{array}$ & $\begin{array}{l}0.35 \\
(0.22-0.51)\end{array}$ & $\begin{array}{l}0.18 \\
(0.00-0.62)\end{array}$ & - & - & - & - & $\begin{array}{l}-10014.6 \\
\end{array}$ & 3.87 & 1 & $\begin{array}{l}\text { Compare CE, } \\
p=0.05\end{array}$ & $20,037.10$ \\
\hline ACE & $\begin{array}{l}0.03 \\
(0.03-0.04)\end{array}$ & $\begin{array}{l}0.03 \\
(0.03-0.04)\end{array}$ & $\begin{array}{l}0.79 \\
(0.59-0.90)\end{array}$ & $\begin{array}{l}0.70 \\
(0.50-0.83)\end{array}$ & $\begin{array}{l}0.44 \\
(0.28-0.62)\end{array}$ & $\begin{array}{l}0.35 \\
(0.22-0.51)\end{array}$ & $\begin{array}{l}0.18 \\
(0.0-0.62)\end{array}$ & $\begin{array}{l}0.18 \\
(0.0-0.62)\end{array}$ & - & $\begin{array}{l}0.61 \\
(0.25-0.97)\end{array}$ & $\begin{array}{l}0.21 \\
(0.06-0.36)\end{array}$ & -10014.5 & 44.6 & 1 & $\begin{array}{l}\text { Compare AE, } \\
p<0.0001\end{array}$ & $20,037.10$ \\
\hline $\mathrm{ADE}$ & $\begin{array}{l}0.03 \\
(0.03-0.04)\end{array}$ & $\begin{array}{l}0.03 \\
(0.03-0.04)\end{array}$ & $\begin{array}{l}0.83 \\
(0.68-0.91)\end{array}$ & $\begin{array}{l}0.41 \\
(0.36-0.47)\end{array}$ & $\begin{array}{l}0.49 \\
(0.35-0.64)\end{array}$ & $\begin{array}{l}0.16 \\
(0.13-0.19)\end{array}$ & $\begin{array}{l}0.83 \\
(0.72-0.94)\end{array}$ & $\begin{array}{l}0.83 \\
(0.72-0.94)\end{array}$ & 0 & - & $\begin{array}{l}0.17 \\
(0.06-0.28)\end{array}$ & -10036.8 & 0 & 1 & $\begin{array}{l}\text { Compare AE, } \\
p=1.0\end{array}$ & $20,081.66$ \\
\hline $\mathrm{AE}$ & $\begin{array}{l}0.03 \\
(0.03-0.04)\end{array}$ & $\begin{array}{l}0.03 \\
(0.03-0.04)\end{array}$ & $\begin{array}{l}0.83 \\
(0.68-0.91)\end{array}$ & $\begin{array}{l}0.41 \\
(0.36-0.47)\end{array}$ & $\begin{array}{l}0.49 \\
(0.35-0.64)\end{array}$ & $\begin{array}{l}0.16 \\
(0.13-0.19)\end{array}$ & $\begin{array}{l}0.83 \\
(0.72-0.94)\end{array}$ & $\begin{array}{l}0.83 \\
(0.72-0.94)\end{array}$ & - & - & $\begin{array}{l}0.17 \\
(0.06-0.28)\end{array}$ & -10036.8 & 34.3 & 1 & $\begin{array}{l}\text { Compared ACE, } \\
p<0.0001\end{array}$ & $20,079.66$ \\
\hline CE & $\begin{array}{l}0.03 \\
(0.03-0.04)\end{array}$ & $\begin{array}{l}0.03 \\
(0.03-0.04)\end{array}$ & $\begin{array}{l}0.74 \\
(0.61-0.83)\end{array}$ & $\begin{array}{l}0.74 \\
(0.61-0.83)\end{array}$ & $\begin{array}{l}0.39 \\
(0.29-0.51)\end{array}$ & $\begin{array}{l}0.39 \\
(0.29-0.51)\end{array}$ & - & - & - & $\begin{array}{l}0.74 \\
(0.63-0.85)\end{array}$ & $\begin{array}{l}0.26 \\
(0.15-0.37)\end{array}$ & -10016.5 & 3.87 & 1 & $\begin{array}{l}\text { Compare ACE, } \\
p=0.05\end{array}$ & $20,038.97$ \\
\hline DE & $\begin{array}{l}0.03 \\
(0.03-0.04)\end{array}$ & $\begin{array}{l}0.03 \\
(0.03-0.04)\end{array}$ & $\begin{array}{l}0.82 \\
(0.66-0.91)\end{array}$ & $\begin{array}{l}0.20 \\
(0.17-0.23)\end{array}$ & $\begin{array}{l}0.48 \\
(0.33-0.63)\end{array}$ & $\begin{array}{l}0.08 \\
(0.07-0.09)\end{array}$ & $\begin{array}{l}0.81 \\
(0.70-0.94)\end{array}$ & - & $\begin{array}{l}0.82 \\
(0.70-0.94)\end{array}$ & - & $\begin{array}{l}0.18 \\
(0.06-0.30)\end{array}$ & -10067.7 & 61.7 & 1 & $\begin{array}{l}\text { Compare ADE, } \\
p<0.0001\end{array}$ & $20,141.35$ \\
\hline
\end{tabular}

$A$ additive genetic, AIC Akaike's Information Criterion, $C$ common environment, $c w C$ case-wise concordance rate, $D$ dominant genetic, $d f$ degrees of freedom, $D Z$ dizygotic, $E$ unique environment, $H$ broad sense heritability, $L L=\log$ likelihood of model, $-2 \mathrm{ln}=$ Likelihood Ratio chi-square test, $M Z$ monozygotic, rho tetrachoric correlation coefficient, Sat saturated model.

The ACE model in bold displayes the best model fit by the AIC. Fixing the common environmental component C at zero produced a significantly worse fit $(p<0.0001)$. 


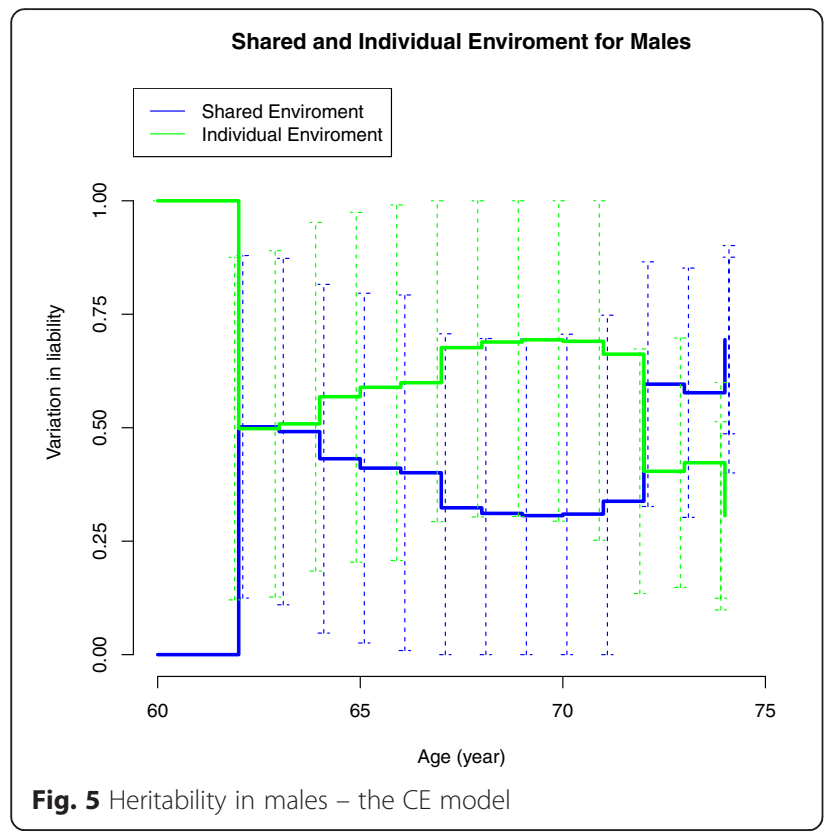

In women an additive genetic influence was detectable, accounting for $6 \%$ of the variation in population liability for knee OA leading to TKA. However, a very interesting finding was the robust significance of the common environmental component in both the sex-stratified and sexadjusted analyses. In terms of family factors, comprising common genes and common environment, $79 \%$ of the variation in liability to knee OA in females was attributable to these factors; however, $69 \%$ of the variation in liability to knee OA in males was attributable to common environmental factors alone. In our sex adjusted analysis the additive genetic component accounted for $18 \%$ of the

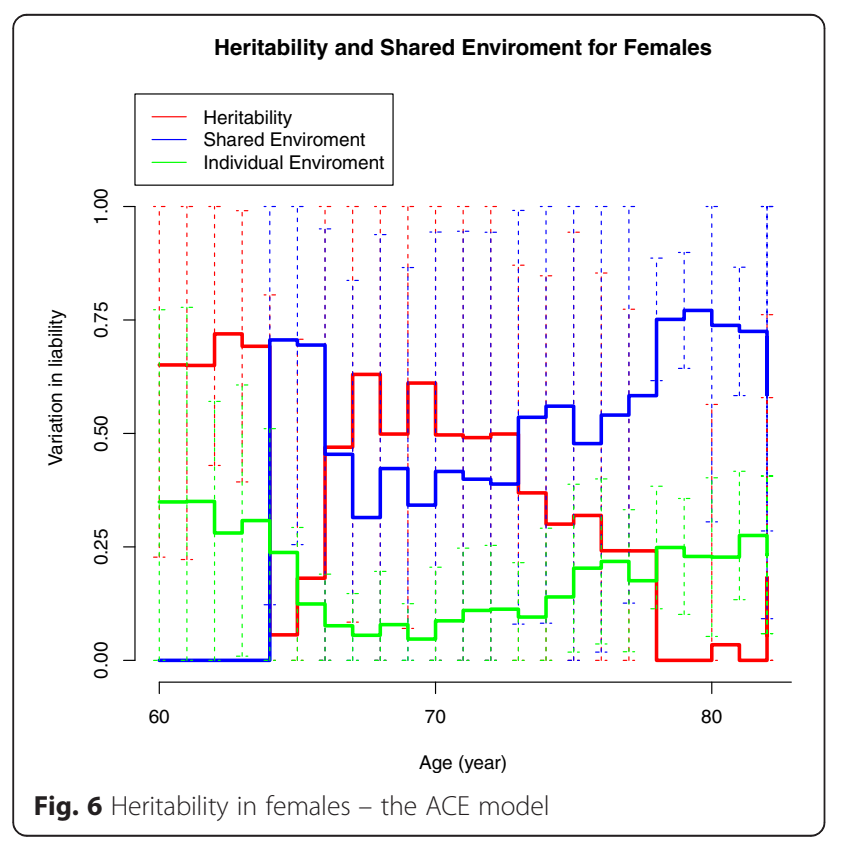

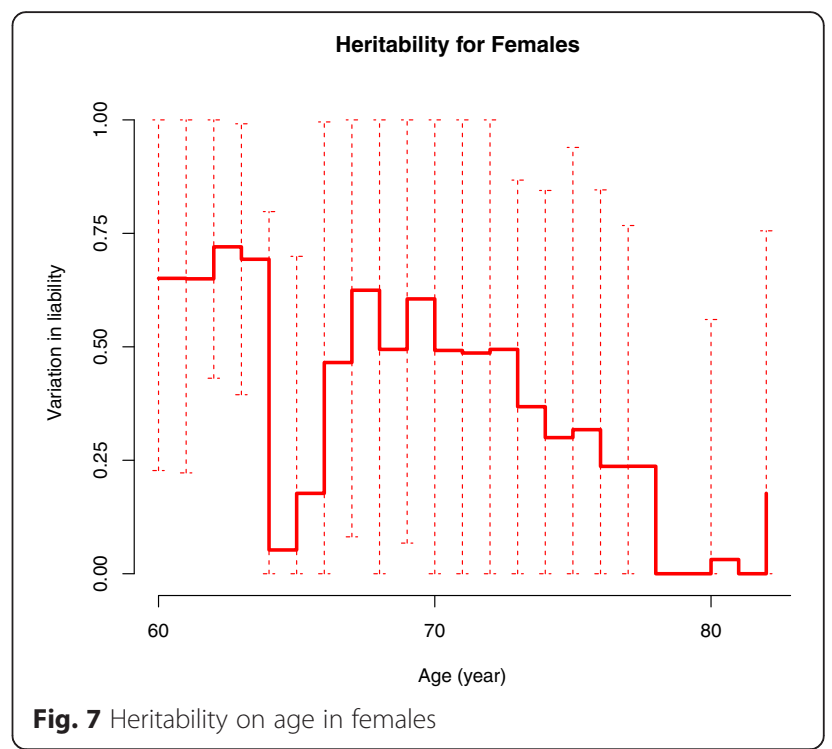

variation in the population liability to TKA due to primary OA leaving $82 \%$ of this variation attributable to environmental factors attributable to $82 \%$ of the variation in population liability for TKA due to primary OA. Consequently, the main findings in our study are that in both males and females knee OA is primarily an environmental disease and the impact of genetic factors is of a lesser significance.

\section{Strengths}

Our study has several strengths. Overall, twins are representative of the general population with respect to common multifactorial complex diseases [38]. Our results support this notion because we found the same distribution of sex and cases in twins as in non-twins in the DKR; $85 \%$ of the twins had undergone a TKA compared with $82 \%$ in the non-twin register population, and a samesex distribution of 61-62\% females occurred in twin and non-twin subpopulations, respectively. Our data were collected from two Danish nationwide and population-based registers including a large twin population comprising all twins alive in 1997 and a follow-up term of 13 years. The completeness of in the DKR is approximately 90-92\% based on annual reports [26]. The DKR has been validated and found to provide a sound basis for large populationbased epidemiological studies [27]. Further, the zygosity misclassification in the DTR of the same-sex twins is an acceptable $5 \%$ [25].

By implementing the CIF we took into account that the occurrence of death and TKA due to primary OA by increasing age are competing events which might otherwise have biased our estimates if not adjusted for by overestimating the number of cases [32].

The cumulative incidence curves reflect the risk of TKA, separately in males and females, and are intuitively 
appealing and easy to understand. Further, the censored nature of the data collected from 1997 to 2010 was accounted for by applying a liability threshold model with right censoring. We defined a case as a twin who had undergone a TKA due to knee OA independent of co-twin status, because these patients represent a welldefined outcome contrary to cases based on conventional radiographic examination with or without symptoms [22]; studies defining knee OA cases from radiographic findings may encounter some difficulties in defining their cases as disease severity varies and the correlation between symptoms or clinical presentation and radiographic findings generally is poor $[6-8,22,39]$. Further, TKA is frequently the treatment of choice when symptoms cannot be controlled in any other way regardless of radiographic findings.

\section{Limitations}

Our study has some important limitations. Some misclassification with respect to the proper diagnosis and zygosity may occur, but a certain misclassification of the diagnosis would be equally distributed between zygosity groups and would not have any predestined pattern (i.e. MZ twin pairs). The principle in the CTD is that if a larger phenotypic similarity is observed in MZ twin pairs compared with that of $\mathrm{DZ}$ twin pairs, a genetic influence on the disease in question can be inferred; however, we consider it unlikely that either misclassification or completeness should be subject to zygosity favouritism, hence this misclassification would be of the non-differential type. Adjustments for confounder effects in register-based studies are only feasible if the relevant information is included in the register in question. In this study we could not adjust for BMI and occupational exposures, as the two registers did not provide this information. However, studies on BMI as a risk factor for knee OA are numerous and conclusive $[9,10]$. Our heritability estimates may be biased because we could not adjust for BMI, in particular whether BMI is considered to be influenced by shared environmental factors. The common environment includes shared family life with respect to upbringing and cohabitation, potentially inflicting a negative association between family factors and the risk of knee OA in later life. BMI is a highly heritable condition [40]; however, common environmental factors have previously been reported to influence BMI significantly in twin studies with sufficient sample size, pointing at a genotype $\times$ environment covariance $\left(\mathrm{CV}_{\mathrm{GE}}\right)[41,42]$. Regarding the high correlation pattern between $\mathrm{BMI}$ and knee $\mathrm{OA}$, a $\mathrm{CV}_{\mathrm{GE}}$ between knee OA susceptibility genes and common environmental influences on BMI is a likely assumption. Such a $\mathrm{CV}_{\mathrm{GE}}$ relationship driven by shared environmental factors may mimic a common environmental influence at the expense of the additive genetic component. $\mathrm{A} \mathrm{CV}_{\mathrm{GE}}$ is a well appreciated bias in the CTD, as well as the difficulties in separating the common environmental component from an additive genetic effect, and is described in detail by Coventry and Keller [43, 44]. If parental phenotypes, more or less of genetic origin, modify the environment of their child or children, a $\mathrm{CV}_{\mathrm{GE}}$ may be present. Consequently, the presence of a $\mathrm{CV}_{\mathrm{GE}}$ will increase the $\mathrm{DZ}$ similarity relative to the $\mathrm{MZ}$ similarity and mimic a common environmental influence at the expense of an additive genetic influence, violating the equal environment assumption essential in the CTD $[43,44]$. Hence, parental BMI susceptibility genes may rule or govern environmental exposure like diet and physical activity preferences, so that throughout life individuals at risk of knee OA may live in high calorie intake and low physical activity environments as they hang on to environments correlated with their genetic propensities [42]. Indeed, in a recent study by Reyes et al. [45], individuals from the lowest socioeconomic status families had a significantly higher risk of knee OA, obesity accounting for $50 \%$ of the excess risk. However, a weakness in the CTD is that it cannot discriminate the various sources of the shared environment, but for this purpose the extended twin-family design is well appreciated [28, 43, 44].

Studies on occupation and occupational exposure to knee-straining work tasks and the risk of symptomatic knee OA have primarily demonstrated a moderate association between kneeling work tasks and heavy lifting, and certain professions (i.e. farming and fishing) as risk factors for knee OA, but primarily in males [14-16, 22]. However, a recent study by Andersen et al. [46] demonstrated an increased risk of knee OA in female health care assistants.

\section{Context}

In our sex-adjusted ACE model (censoring included) a heritability estimate of $18 \%(0 ; 62)$ and a common environmental component of $61 \%(25 ; 97)$ were found. To our knowledge, this is the first nationwide population-based twin study demonstrating a highly significant influence from common environmental factors in the liability for TKA due to primary knee OA. Few twin studies on knee OA have been published; however, our study indicates a non-significant additive genetic component of $6 \%$ in women, which is much lower than the findings of Spector et al. [17] and MacGregor et al. [18] of $39 \%$ and $37 \%$ respectively. These latter studies were both cross-sectional in design and based on women with radiographic knee OA, a much younger population compared with our study. By design, these studies could not take into account the competing event of death that may influence the age-related occurrence of symptomatic knee OA and the resulting heritability estimates. Our lower estimate may partly be a consequence of not being able to adjust for confounders such as BMI, but our graphical presentation 
in Fig. 6 of the heritability in the ACE model in females indicates a higher genetic component in younger females, decreasing with increasing age to disappear at age around 80 years. In Fig. 7 the heritability on age in females is displayed with an estimate of $40 \%$ at age 65 . The drop in heritability at ages 64-65 years does not reflect a biological plausible course, but rather is caused by the difficulties in the CTD of separating the additive genetic component from that of the common environment as described previously.

That genetic influences on a trait may change with age has recently been described in a large twin-based follow-up study where the age-associated changes in bone mineral density were reported to be highly heritable in younger women, but the heritability decreased by increasing age to disappear after the age of 65 years [47]. In the sibling study on knee OA by Neame et al. [20] a heritability estimate of $62 \%$ was reported, although the authors did recommend a cautious interpretation as they could only adjust for age.

We could not detect a genetic component in males. This is in line with the twin study by Kujala et al. [21] on hip, knee, hand and ankle OA, which failed to establish a genetic component in males. We have recently examined the risk and heritability of primary hip OA leading to total hip arthroplasty, implementing the same methodology as in the present study. We found a highly significant additive genetic component after adjustment for sex of $47 \%$ and a common environmental component of $22 \%$ of the variation in liability for total hip arthroplasty due to primary hip OA [48]. These findings indicate that the genetic and environmental influences differ by joint site and support the findings by MacGregor et al. [18] that hip and knee OA share less than $1 \%$ common genes and that genetic and environmental factors impact differently in the two weight-bearing joints.

Knee OA pain may differ by sex, in that females tend to report higher pain score compared with males and a variety of socio-economic and mental factors may interact with knee OA pain $[49,50]$. In our study, data on pain score, socio-economic factors or mental complaints were not available, leaving no possibility to adjust for these potential confounders or to examine a genetic influence on knee OA pain per se.

However, by including both MZ and SS-DZ as well as OS-DZ twin pairs, adjustment for gender was feasible and our heritability estimate reflects the influence from genetic, common and environmental factors on end-stage knee OA leading to TKA after adjustment for sex effects.

\section{Implications}

The graphical presentation of the cumulative incidence provides a ready instrument assessing the risk of TKA and provides helpful insight to clinicians as well as to health care planners and occupational hygienists, because our study indicates that the influence from environmental factors on TKA risk is highly important in both genders. In western societies the populations are getting older and the proportion of older individuals is increasing rapidly, as is overweight and obesity. These factors increase the risk of knee OA, increase the health economic burden and increase the risk of death [1-5]. This development will need increasing focus and our study provides some evidence that intervention by clinical and public health measures, including preventive measures, should focus in particular on the family from childhood and adolescence to prevent increasing knee $\mathrm{OA}$ incidence and prevalence.

\section{Conclusion}

Our study indicates that the impact of genetic factors on knee OA leading to TKA differ in females and males, but simultaneously also demonstrates the presence of a highly significant influence from common and unique environmental factors. In the sex-stratified model fitting, an additive genetic influence of $6 \%$ in females was detected, but in males no genetic component was found. However, after sex adjustment the impact of environmental factors remained highly significant, leaving a moderate additive genetic influence of $18 \%$ in the population liability for TKA. Further studies focusing on the discrimination of the various sources of the common environment in exposed families and individuals are needed, as well as studies on preventive measures.

\section{Data sharing}

None.

\section{Abbreviations}

A: Additive genetic; AlC: Akaike's Information Criterion; BMI: Body mass index; $\mathrm{C}$ : Common environment; $\mathrm{Cl}$ : Confidence interval; CIF: Cumulative incidence function; CTD: Classical twin design; $\mathrm{CV}_{\mathrm{GE}}$ : genotype $\times$ environment covariance; D: Dominant genetic; DKR: Danish Knee Arthroplasty Register; DTR: Danish Twin Register; DZ: Dizygotic; E: Unique environment; IPW: Inverse probability weighting; MZ: Monozygotic; M17.0: Primary gonarthrosis, bilateral; M17.1: Other primary gonarthrosis; OA: Osteoarthritis; OS-DZ: Opposite-sex dizygotic; rho: tetrachoric correlation coefficient; SS-DZ: Same-sex dizygotic; TKA: Total knee arthroplasty; ICD10: WHO International Classification of Diseases version 10.

\section{Competing interests}

All authors have completed the Unified Competing Interest Form online (http://www.icmje.org/about-icmje/faqs/conflict-of-interest-disclosure-forms) (available on request from the corresponding author) and declare: no support from any organisation for the submitted work; no financial relationships with any organisations that might have an interest in the submitted work in the previous three years; and no other relationships or activities that could appear to have influenced the submitted work.

\section{Authors' contributions}

SGS developed the idea and the project, performed statistical analysis and wrote the manuscript, revisions and final paper. AS participated in the project development, provided the data from the DTR and data maintenance, applied valuable criticism, and revised and accepted the final paper. SM developed the statistical approach based on the idea and calculated statistical analysis, and revised and accepted the final paper. SO participated in the development, applied valuable criticism to the statistical 
approach and results, and revised and accepted the final paper. LPAB participated in the development of the idea and project, applied valuable criticism, and revised and accepted the final paper. All authors read and approved the final manuscript.

\section{Acknowledgements}

The authors thank Dr Alma Pedersen, MD, PhD, for providing the data from the DKR, and also the DTR for access to the twin data.

\section{Funding}

This study was funded by The Danish Rheumatism Association, and part of SGS's PhD project was funded by the same organization.

\section{Author details}

${ }^{1}$ Department of Occupational and Environmental Medicine, Odense University Hospital, 5000 Odense C, Denmark. ²Department of Orthopaedic Surgery and Traumatology \& Orthopedic Research Unit, Institute of Clinical Research, University of Southern Denmark, 5000 Odense C, Denmark ${ }^{3}$ Clinical Institute, University of Southern Denmark, 5000 Odense C, Denmark. ${ }^{4}$ Department of Epidemiology, Biostatistics and Biodemography, The Danish Twin Registry, University of Southern Denmark, 5000 Odense C, Denmark.

Received: 19 November 2015 Accepted: 19 January 2016

\section{Published online: 11 February 2016}

\section{References}

1. WHO Scientific Group on the Burden of Musculoskeletal on conditions of the Start of the New Millennium. The burden of musculoskeletal conditions at the start of the new millennium. World Health Organ Tech Rep Ser. 2003;919:i-x. 1-218.

2. Woolf AD, Pfleger B. Burden of major musculoskeletal conditions. Bull World Health Organ. 2003;81:646-56.

3. Culliford D, Maskell J, Judge A, Cooper C, Prieto-Alhambra D, Arden NK Future projections of total hip and knee arthroplasty in the UK: results from the UK Clinical Practice Research Datalink. Osteoarthritis Cartilage. 2015;24:594-600

4. Wilkie R, Phillipson C, Hay EM, Pransky G. Anticipated significant work limitation in primary care consulters with osteoarthritis: a prospective cohort study. BMJ Open. 2014;4:e005221.

5. Nuesch E, Dieppe P, Reichenbach S, Williams S, Iff S, Juni P. All cause and disease specific mortality in patients with hip and knee osteoarthritis: population based cohort study. BMJ. 2011;342:d1165.

6. Felson DT, Naimark A, Anderson J, Kazis L, Castelli W, Meenan RF. The prevalence of knee osteoarthritis in the elderly. Arthritis Rheum. 1987:30:914-8.

7. Felson DT, Zhang Y, Hannan MT, Naimark A, Weisman BN, Aliabadi P, et al. The incidence and natural history of knee osteoarthritis in the elderly. Arthritis Rheum. 1995;38:1500-5.

8. Felson DT, Zhang Y. An update on the epidemiology of knee and hip osteoarthritis with a view to prevention. Arthritis Rheum. 1998;41:1343-55.

9. Murphy L, Schwartz TA, Helmick CG, Renner JB, Tudor G, Koch G, et al. Lifetime risk of symptomatic knee osteoarthritis. Arthritis Rheum. 2008;59:1207-13.

10. Manek NJ, Hart D, Spector TD, MacGregor AJ. The association of body mass index and osteoarthritis of the knee joint. An examination of genetic and environmental influences. Arthritis Rheum. 2003;48:1024-9.

11. Wills AK, Black S, Cooper R, Coppack RJ, Hardy R, Martin KR, et al. Life course body mass index and risk of knee osteoarthritis at the age of 53 years: evidence from the 1946 British birth cohort study. Ann Rheum Dis. 2012;71:655-60.

12. Roos EM. Joint injury causes knee osteoarthritis in young adults. Curr Opin Rheumatol. 2005;17:195-200

13. Thelin N, Holmberg S, Thelin A. Knee injuries account for the sports-related risk of knee osteoarthritis. Scand J Med Sci Sports. 2006;16:329-33.

14. Lohmander LS, Englund PM, Dahl LL, Roos EM. The long-term consequences of anterior ligament and meniscus injuries: osteoarthritis. Am J Sports Med. 2007;35:1756-69.

15. Jensen LK. Knee osteoarthritis: influence of work involving heavy lifting, kneeling, climbing stairs or ladders, or kneeling/squatting combined with heavy lifting. Occ Environ Med. 2008;65:72-89.

16. Coggon D, Croft P, Kellingray S, Barrett D, McLaren M, Cooper C. Occupational physical activities and osteoarthritis of the knee. Arthr Rheum. 2000;43:1443-9.
17. Spector TD, Cicuttini F, Baker J, Loughlin J, Hart D. Genetic influence on osteoarthritis in women: a twin study. BMJ. 1996:312:940-3.

18. MacGregor AJ, Li Q, Spector TD, Williams FMK. The genetic influence on radiographic osteoarthritis is site specific at the hand, hip and knee. Rheumatol. 2009:48:277-80.

19. Zhai G, Hart DJ, Kato BS, MacGregor A, Spector TD. Genetic influence on the progression of radiographic knee osteoarthritis: a longitudinal twin study. Osteoarthritis Cartilage. 2007;15:222-5.

20. Neame RL, Muir K, Doherty S, Doherty M. Genetic risk of knee osteoarthritis: a sibling study. Ann Rheum Dis. 2004;63:1022-7.

21. Kujala UM, Leppaevouri J, Kaprio J, Kinnunen J, Peltonen L, Kuskenvuo M. Joint-specific twin and familial aggregation of recalled physician diagnosed osteoarthritis. Twin Res. 1999:2:196-202.

22. Franklin J, Ingvarsson T, Englund M, Lohmander S. Association between occupation and knee and hip replacement due to osteoarthritis: a casecontrol study. Arthritis Res Ther. 2010;12:R102.

23. Skytthe A, Kyvik K, Holm NV, Vaupel JW, Christensen K. The Danish Twin Registry: 127 birth cohorts of twins. Twin Res. 2002;5:352-7.

24. Skytthe A, Kyvik K, Bathum L, Holm N, Vaupel JW, Christensen K. The Danish Twin Registry in the new millennium. Twin Res Hum Genet. 2006;9:763-71.

25. Christiansen L, Frederiksen H, Schousboe K, Skytthe A, von Wurmb-Schwark $\mathrm{N}$, Christensen $\mathrm{K}$, et al. Age- and sex-differences in the validity of questionnaire-based zygosity in twins. Twin Res. 2003;6:275-8.

26. Dansk Knæalloplastik Register. Annual report 2011, 2012, 2013. http://www. kea.au.dk/da/kliniskkvalitet/kliniske-databaser/KnaealloplastikRegister.html. Last annual report was accessed by: https://www.sundhed.dk/content/cms/ 99/4699_dkr_2013.pdf.

27. Pedersen AB, Mehnert F, Odgaard A, Schrøder HM. Existing data sources for clinical epidemiology: The Danish Knee Arthroplasty Register. Clin Epidemiol. 2012;4:125-35.

28. Neale MC, Cardon LR. Methodology for genetic studies of twins and families. Berlin: Kluwer Academic Publishers; 1992.

29. Falconer DS. The inheritance of liability to diseases with variable age of onset, with particular reference to diabetes mellitus. Ann Hum Genet. 1967;31:1-20.

30. Haesook TK. Cumulative incidence in competing risks data and competing risks regression analysis. Clin Cancer Res. 2007;13.

31. Gillam MH, Ryan P, Graves SE, Miller NM, de Steiger RN, Salter A. Competing risks survival analysis applied to data from the Australian Orthopaedic National Joint Replacement Registry. Acta Orthopaed. 2010;81:548-55.

32. Gillam MH, Salter A, Ryan P, Graves SE. Different competing risks models applied to data from the Australian Orthopaedic Association National Joint Replacement Registry. Acta Orthopaed. 2011;82:513-20.

33. Ørnulf B. Aalen-Johansen Estimator. Encyclopedia of Biostatistics; 2005. doi:10.1002/04700/11815.b2a1101.

34. Scheike TH, Holst K, Hjelmborg J. Estimating heritability for cause specific mortality based on twin studies. Lifetime Data Anal. 2013;20(2): 210-33. doi:10.1007/s10985-013-9244-x.

35. Samore MH, Shen S, Green T, Stoddard G, Saur B, Shinogle J, et al. A Simulation-based evaluation of methods to estimate the impact of an adverse event on hospital length of stay. Med Care. 2007;45:S108-15.

36. Hougaard P. Fundamentals of survival data. Biometrics. 1999:5:13-22.

37. Scheike $T H$, Holst $K$, Hjelmborg J. Estimating the concordance function for bivariate competing risks data. Stat Med. 2014;33:1193-204.

38. Andrew T, Hart DJ, Sneider H, de Lange M, Spector TD, MacGregor AJ. Are twins and singletons comparable? A study of disease-related and lifestyle characteristics in adult women. Twin Res. 2001;4:464-77.

39. Guermazi A, Niu J, Hayashi D, Englund M, Neogi T, Aliabadi P, et al. Prevalence of abnormalities in knees detected by MRI in adults without knee osteoarthritis: population based observational study (Framingham Osteoarthritis Study). BMJ. 2012:345:e5339.

40. Haworth CMA, Carnell S, Meaburn LE, Davis OSP, Plumin R, Wardle J. Increasing heritability of BMI and stronger associations with the FTO gene over childhood. Obesity. 2008;16:2663-8.

41. Silventoinen K, Jaakko Kaprio J. Genetics of tracking of body mass index from birth to late middle age: evidence from twin and family studies. Obes Facts. 2009:3:196-202

42. Silventoinen K, Rokholm B, Kaprio J, Sørensen TIA. The genetic and environmental influences on childhood obesity: a systematic review of twin and adoption studies. Int J Obes. 2010;34:29-40. 
43. Coventry WL, Keller MC. Estimating the extent of parameter bias in the classical twin design: a comparison of parameter estimates from extended twin family and classical twin design. Twin Res Hum Gen. 2005;8:214-23.

44. Keller MC, Coventry WL. Quantifying and addressing parameter indeterminacy in the classical twin design. Twin Res Hum Gen. 2005;8:201-13.

45. Reyes C, Garcia-Gil M, Elorza JM, Mendez-Boo L, Hermosialla E, Javaid MK, et al. Socio-economic status and the risk of developing hand, hip or knee osteoarthritis: a region-wide ecological study. Osteoarthritis Cartilage. 2015 23(8):1323-9. doi:10.1016/j.joca.2015.03.020

46. Andersen S, Thygesen LC, Davidsen M, Helweg-Larsen K. Cumulative years in occupation and the risk of hip or knee osteoarthritis in men and women: a register-based follow-up study. Occup Environ Med. 2012;69:325-30.

47. Moayyeri A, Hammond CJ, Hart DJ, Spector TD. Effects of age on genetic influence on bone loss over 17 years in women: The Healthy Ageing Twin Study (HATS). J Bone Miner Res. 2012:27:2170-8.

48. Skousgaard SG, Möller S, Skytthe A, Brandt LPA, Overgaard S. Probability and heritability estimates on primary osteoarthritis of the hip leading to total hip arthroplasty: a nationwide population based follow-up study in Danish twins. Arthritis Res Ther. 2015;17:336.

49. Feldman CH, Dong Y, Katz JN, Donnell-Fink LA, Losina E. Association between socioeconomic status and pain, function and pain catastrophizing at presentation for total knee arthroplasty. BMC Musculoskel Dis. 2015;16(1):18. doi:10.1186/s12891-015-0475-8.

50. Jämsen E, Jäntti P, Puolakka T, Eskelinen A. Primary knee replacement for primary osteoarthritis in the aged: gender differences in epidemiology and preoperative clinical state. Aging Clin Exp Res. 2012;24:691-8.

\section{Submit your next manuscript to BioMed Central} and we will help you at every step:

- We accept pre-submission inquiries

- Our selector tool helps you to find the most relevant journal

- We provide round the clock customer support

- Convenient online submission

- Thorough peer review

- Inclusion in PubMed and all major indexing services

- Maximum visibility for your research

Submit your manuscript at www.biomedcentral.com/submit

C Biomed Central 